\title{
Structural Switch of AIN Sputtered Thin Films From (101) to (002) Orientation, Driven by the Growth Kinetics
}

\author{
A.Taurino $^{1}$, M.A. Signore ${ }^{1}$, M. Catalano ${ }^{1,2}$ and M. J. Kim ${ }^{2}$ \\ 1. CNR IMM, Institute for Microelectronics and Microsystems, Via Monteroni, Lecce, Italy \\ 2. Dep. of Materials Science and Engineering, Univ. of Texas at Dallas, Richardson, Texas, USA
}

Due to its wide bandgap, high thermal conductivity, high electrical resistivity, temperature independent piezoelectric coefficient, aluminum nitride (AIN) represents a material of great technological interest, for applications in RF MEMS devices, high power electronics, energy harvesting and optoelectronics [1]. In the specific case of piezoelectric applications, the performances of the device strongly depend on the AlN microstructural properties, because AIN thin films grown along c-axis orientation exhibit intense piezoelectric response, the (001) orientation having the highest piezoelectric constants [2].

In this work, AlN thin films were deposited by RF magnetron sputtering on $\mathrm{Si} / \mathrm{SiO}_{2}$ substrate, by using an aluminum target, 99.999\%-pure. A Ti underlayer, $100 \mathrm{~nm}$-thick, was sputtered on the substrate as seed layer for the growth of the nitride [3]. The RF power applied to the Al target was $150 \mathrm{~W}$ and the $\mathrm{N}_{2}$ flux percentage was set at $60 \%$; the total chamber pressure was tuned by the throttle valve between $2.5 \times 10^{-2}$ mbar and $4.0 \times 10^{-3}$ mbar. The substrate was not heated during the growth process. The films crystalline structure was analyzed by X-ray diffraction, using $\mathrm{Cu}-\mathrm{K} \alpha$ radiation in the $\theta-2 \theta$ configuration. Transmission Electron Microscopy (TEM) and Scanning Transmission Electron Microscopy (STEM) analyses were performed on cross-sectional TEM lamellas prepared by Focused Ion Beam (FIB) in a FEI NOVA 200 Cross Beam Focused Ion Beam. The investigations were carried out by using a JEMARM200F TEM/STEM instrument, operating at $200 \mathrm{kV}$, with a resolution of $78 \mathrm{pm}$.

Figure 1 shows the XRD spectra obtained from the samples grown at $2.5 \times 10^{-2}$ mbar and $4.0 \times 10^{-3}$ mbar. The spectra evidence the presence of (002) and (100) Ti peaks for both films, whose intensity ratio is comparable, whereas, for the AlN films, the preferential (002) orientation obtained at lower pressure switches to (101) at higher pressure. As shown in figure 2, The high angle annular dark field (HAADF) $(a, e)$ and bright field (BF) (b, f) STEM images of the two samples demonstrate the regularity of the films thickness and interfaces and their columnar organization, as also evidenced by the TEM dark field (DF) images (c, g), obtained by selecting the diffraction maxima labelled in the diffraction patterns (DP) $(d, h)$. These give further indication about the orientation of the films, which result to be polycrystalline and textured. Table I reports the lattice distances inferred from the analysis of the diffraction patterns. In the case of the film grown at lower pressure, two intense diffraction maxima (in the circle) occur in the pattern corresponding to the (002) AlN and Ti planes. These maxima are aligned along the same direction, perpendicular to the substrate, indicating the (002) preferential growth. For the film deposited at higher pressure, the same periodicities are obtained, but the AIN film is now preferentially oriented along the (101) direction (diffraction maxima in the circle).

These results can be interpreted on the base of simple considerations related to the growth kinetics. The growth kinetics influences the film crystalline organization, and is determined by the deposition parameters controlling the energy of the species impinging on the substrate (substrate bias voltage and temperature and total pressure in the deposition chamber). The structural analyses discussed above demonstrates that the decrease in the total pressure by one order of magnitude leads to a structural 
switch from (101) to (002) orientation. A decrease in the pressure means an increase in the mean free path (MFP) of the sputtered atoms ( $\mathrm{Al}$ and $\mathrm{N}$ ), which reach the substrate with higher energies, favoring the proper (002) planes arrangement. According to the MPF formula, lower gas pressures correspond to larger MPF and to a lower number of collisions. As a consequence, the sputtered atoms will keep a significant part of their initial kinetic energy when arriving at the substrate surface; this enhances the adatom mobility towards the low energy lattice sites, favoring the growth along the 002 axis. On the contrary, a higher collisions number leads to Al-N dimers formation which generally arrange along (101) planes.

References:

[1] H Hirayama et al, Phys. Status Solidi A 6 (2009) p. 1176.

[2] J Lin and R Chistyakov, Appl. Surf. Sci. 396 (2017) p.129.

[3] MA Signore et al, Mater. Design 119 (2017) p. 15.

[4] M V Pelegrini et al, J. Phys. 92 (2014) p. 940.

[5] A Luque and A Martì, Phys. Rev. Lett. 78 (1997), p. 5014.

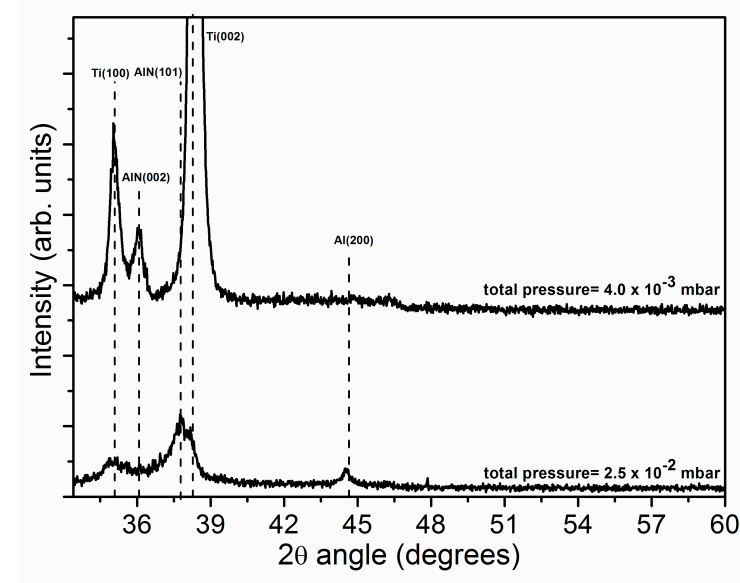

Figure 1. XRD pattern of the AlN samples.

\begin{tabular}{|c|c|c|c|}
\hline $\begin{array}{l}\text { Ring } \\
\text { label }\end{array}$ & $\begin{array}{l}\text { Experimental } \\
\text { lattice } \\
\text { distance }(\AA) \\
\text { (d)/(h) }\end{array}$ & $\begin{array}{l}\text { Ti lattice } \\
\text { distance } \\
(\AA) \\
\text { d(hkl) }\end{array}$ & $\begin{array}{l}\text { AIN lattice } \\
\text { distance }(\AA) \\
\text { d(hkl) }\end{array}$ \\
\hline 1 & $2.68 / 2.69$ & & $2.69(100)$ \\
\hline 2 & $2.50 / 2.48$ & & $2.49(002)$ \\
\hline 3 & $2.36 / 2.35$ & $2.35(002)$ & $2.37(101)$ \\
\hline 4 & 2.23 & $2.24(101)$ & \\
\hline 5 & $1.84 / 1.82$ & & $1.83(012)$ \\
\hline 6 & 1.72 & & $1.73(012)$ \\
\hline 7 & $1.55 / 1.54$ & $1.56(003)$ & $1.55(110)$ \\
\hline 8 & $1.41 / 1.41$ & $1.41(111)$ & $1.41(103)$ \\
\hline
\end{tabular}

Table I. Experimental and theoretical lattice spacings.
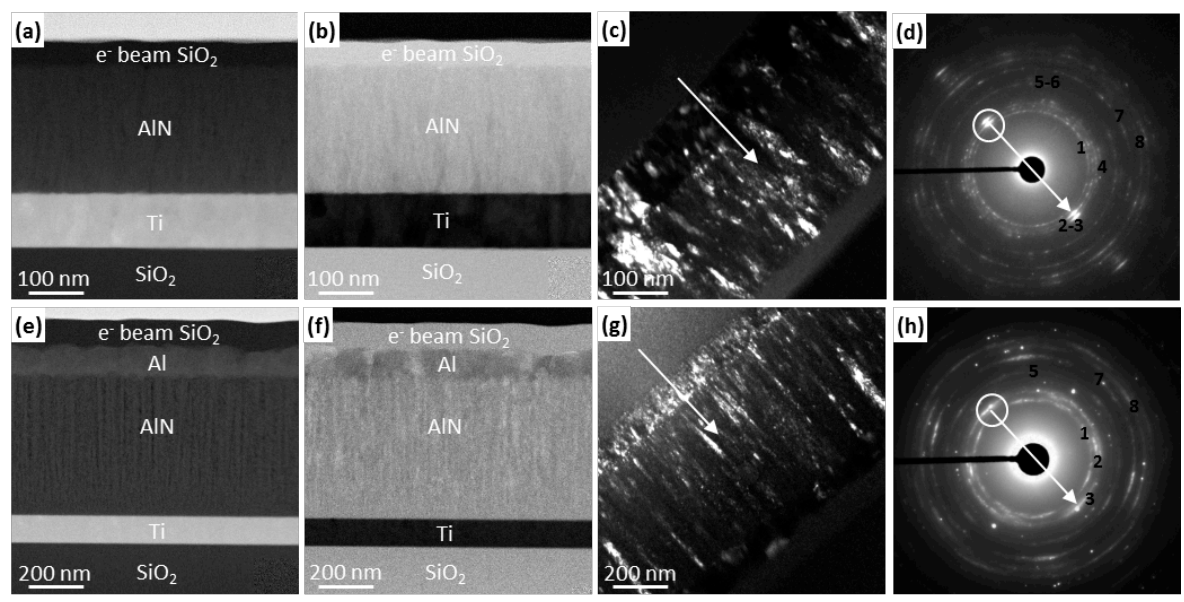

Figure 2. HAADF (a, e) and BF (b, f) STEM images of the AIN samples deposited at low (a-d) and high (e-h) pressure. TEM DF images (c, g) obtained by selecting the diffraction maxima labelled by a circle in the DP $(d, h)$. 